\title{
MATHEMATICAL MODEL OF THE MAGNETIC CIRCUIT OF NEW MAGNETO ELASTIC FORCE SENSORS
}

\author{
Amirov Sulton Fayzullaevich ${ }^{1}$, \\ Juraeva Kamila Komilovna', \\ Slobodan Bojanić ${ }^{2}$
}

${ }^{1}$ Tashkent Institute of Engineers of Railways Transports, Uzbekistan

2Universidad Politecnica de Madrid, Spain
Correspondence:

Slobodan Bojanić

e-mail:

slobodan.bojanc@upm.es

\begin{abstract}
:
The mathematical models of magnetic circuits of the developed magnetoelastic force sensors are developed, taking into account the distributed character of the magnetic resistances of concentric ferromagnetic cores and the magnetic capacitance between these coaxially located cores. It is shown that with an increase in the magnetic flux damping coefficient along the magnetic circuit, the degree of nonlinearity of the change in the magnetic voltage and the inconstancy of the magnetic flux along the magnetic circuit increases.
\end{abstract}

\section{Keywords:}

magnetoelastic sensor, magnetic circuit, magnetic flux, mathematical model, ferromagnetic core, distributed parameter.

\section{INTRADUCTION}

The magnetic circuit of the developed magnetoelastic force sensors (MUD) $[1,2]$ is a chain with distributed magnetic and concentrated electrical parameters $[3,4]$. The distributed parameters include the linear values of the magnetic capacity of the air gap between concentric ferromagnetic closed rings $\left(C_{\mu \mathrm{I}}=\right.$ const $)$ and the magnetic resistance of these ferromagnetic rings $\left(Z_{\mu \mathrm{n}}\right)$ per unit angle of the ferromagnetic rings. The transverse ampere turns $\left(F_{b}\right)$ belong to the concentrated parameter. In contrast to electrical circuits with distributed parameters, long magnetic circuits with distributed parameters are more complex. This is due to the fact that in magnetic circuits of even small linear dimensions (in the order of units of centimeters) a significant part of the magnetic flux branches off from the main circuit in the form of scattering fluxes. In addition, the calculation of magnetic circuits with distributed parameters is significantly complicated by the need to take into account the nonlinear properties of the magnetic core material, the losses due to eddy currents and magnetic hysteresis [4].

In electromagnetic sensors with distributed parameters, which include the forces developed by the MUD, the main factors are the nature of the change in magnetic flux and magnetic stress along the length of the magnetic circuit, and also the nature of the dependence of the change in the magnetic permeability of the magnetic circuit material on the applied mechanical force, i.e. $\mu=f(\sigma)[5]$. Therefore, in practice the analysis of the MMA reduces to an analysis of the distribution of this magnetic flux along the length of the magnetic circuit and the determination of the dependence $\mu=f(\sigma)$. 


\section{MAIN PART}

For a theoretical study of the main characteristics of the developed MUD forces, it is necessary to have analytical expressions for the magnetic flux and magnetic stress as a function of the length of the magnetic circuit with distributed parameters.

In view of the above-mentioned reasons, the apparatus of the theory of chains with distributed parameters seems to be optimal for the investigation of magnetic circuits developed by the MDM. The theory of distributed magnetic circuits makes it possible to obtain convenient comparisons with engineering calculations and at the same time sufficiently accurate results by relatively simple methods [3].

To calculate the magnetic circuits of the MUD with distributed parameters, in which there are no moving parts, the most effective methods are the method of substitution schemes involving computer technology and the classical method of constructing and solving differential equations [6].

In this paper, the calculation of the magnetic circuit developed by the MUD forces [1], whose magnetic system is shown in Fig. 1, we produce by the classical method of compiling and solving differential equations.

In order to simplify the analysis of the magnetic circuit, we neglect lateral scattering fluxes and assume that the ring closed ferromagnetic cores, as well as the ferromagnetic bridges diametrically connecting the coaxially located annular closed ferromagnetic cores, are identical. These assumptions do not introduce tangible inaccuracies in the calculations, but significantly simplify the analysis of the considered circuit.

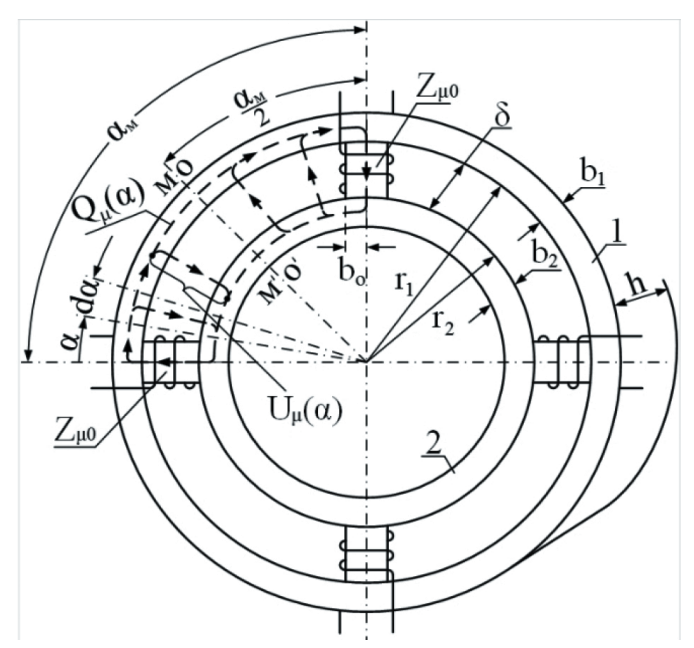

Fig.1. Magnetic system developed by the MUD efforts on [1].
Electromagnetic processes in the magnetic circuit under consideration with distributed parameters are characterized by two integral values: the magnetic flux $Q_{\mu}=(\alpha, t)$ and the magnetic voltage-the difference of the magnetic potentials $U_{\mu}=(\alpha, t)$, which vary both in time $t$ and in coordinate (the length of the chain). The nonlinear and ambiguous properties of ferromagnetic materials make it difficult to analyze the processes in detail in time, so the method of equivalent sinusoids with simultaneous use of complex magnetic conductivity was widely used. In this case, real time functions $Q_{\mu}=(\alpha, t)$, $U_{\mu}=(\alpha, t)$ are replaced by equivalent sinusoidal functions [7], which corresponds to a transition from a real hysteresis loop to an equivalent ellipse. Such a method is physically justified and opens the way for the application of a complex method to nonlinear circuits without a significant loss of accuracy of calculation.

The magnetic system of the developed MUD works with a wide range of magnetic induction variations, therefore, it becomes necessary to take into account the nonlinearity of the main magnetization curve. Nonlinearity will be taken into account by using the dependence of the specific magnetic resistance $\rho_{\mu}$ of steel on magnetic induction $B$ [3].

In the very first approximation, taking into account the nonlinearity of the main magnetization curve, we shall carry out by means of the average value with the help of the average value of the specific magnetic resistance $\rho_{\mu \text { cp }}$, defined in the form

$\rho_{\mu \mathrm{cp}}=\rho_{\mu \min }-\frac{\rho_{\mu \min }-\rho_{\mu \max }}{2}=\frac{\rho_{\mu \min }+\rho_{\mu \max }}{2}$,

Where $\rho_{\mu \min }$ and $\rho_{\mu \max }$ are respectively the minimum and maximum values of the specific magnetic resistance, corresponding to the lower and upper limits of the change in the working magnetic induction.

Based on Kirchhoff's laws, we will derive differential equations for the magnetic flux in closed circular ferromagnetic magnetic cores and the magnetic voltage between them, created by concentrated MDS $F_{b}$, for the elementary section of the magnetic circuit $d \alpha$ (Figures 1 and 2) [3, 4]: 


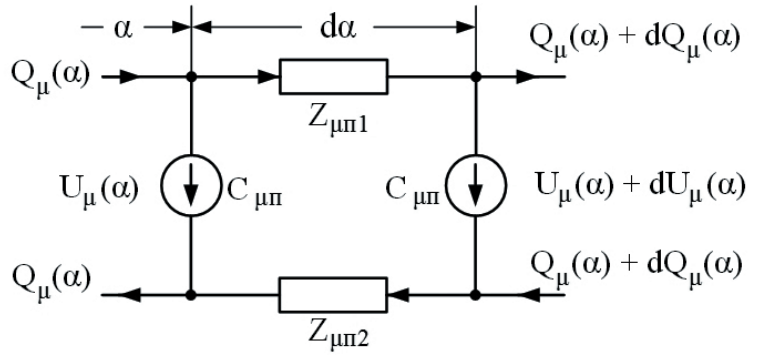

Fig.2. The circuit of substitution of an elementary section of a magnetic circuit $d \alpha$

$Q_{\mu}(\alpha)-U_{\mu}(\alpha) C_{\mu \mathrm{I}} d \alpha-Q_{\mu}(\alpha)-d Q_{\mu}(\alpha)=0$ or

$\frac{d Q_{\mu}(\alpha)}{d \alpha}=-U_{\mu}(\alpha) C_{\mu п}$

$-U_{\mu}(\alpha)+Z_{\mu \mathrm{m} 1} Q_{\mu}(\alpha) d \alpha+U_{\mu}(\alpha)+d U_{\mu}(\alpha)+Z_{\mu \mathrm{m} 2} Q_{\mu}(\alpha) d \alpha=0$

or

$\frac{d U_{\mu}(\alpha)}{d \alpha}=-\left(Z_{\mu \mathrm{m} 1}+Z_{\mu п 2}\right) Q_{\mu}(\alpha)$

where $Q_{\mu}(\alpha), U_{\mu}(\alpha)$ - respectively, the magnetic flux in closed concentric annular cores and the magnetic voltage between; $Z_{\mu \mathrm{m} 1}=\frac{1}{\mu \mu_{0} b_{1}}$ and $Z_{\mu \mathrm{m} 2}=\frac{1}{\mu \mu_{0} b_{2}}$ - The linear values of the magnetic resistances of concentric ferromagnetic cores 1 and 2, per unit magnetic-circuit angle, for convenience of calculation, we take chains $Z_{\mu \mathrm{n} 1}=Z_{\mu \mathrm{n} 2}=Z_{\mu \text { п }}$ $; C_{\mu \mathrm{m}}=\mu_{0} \frac{h}{\delta}$ - The linear value of the magnetic capacitance of the annular gap between the concentric ferromagnetic cores 1 and 2 . The geometric dimensions of the cores 1 and 2 are shown in Fig. 1.

Differentiating (3) and substituting (2) into it, we obtain the following linear homogeneous differential equation of the second order $[3,4]$ :

$\frac{d^{2} U_{\mu}(\alpha)}{d \alpha^{2}}=\left(Z_{\mu \mathrm{m} 1}+Z_{\mu \mathrm{m} 2}\right) C_{\mu \mathrm{I}} U_{\mu}(\alpha)$

The general solution of the differential equation (4) has the following form:

$U_{\mu}(\alpha)=A_{1} e^{\gamma \alpha}+A_{2} e^{-\gamma \alpha}$,

here $\gamma=\sqrt{2 Z_{\mu \mathrm{m}} C_{\mu \mathrm{n}}}$ - Coefficient of magnetic flux propagation along the magnetic circuit, $1 / \mathrm{M} ; A_{1}$ and $A_{2}$ - are integration constants.
Substituting (5) into (3), we obtain

$$
Q_{\mu}(\alpha)=-\frac{\gamma}{2 Z_{\mu \mathrm{\Pi}}} A_{1} e^{\gamma \alpha}+\frac{\gamma}{2 Z_{\mu \mathrm{\Pi}}} A_{2} e^{-\gamma \alpha}
$$

Coefficients $A_{1}$ and $A_{2}$ are determined in this case, based on the following boundary conditions (boundary) conditions:

$$
\left.\begin{array}{l}
\left.U_{\mu}(\alpha)\right|_{\alpha=0}=F_{\theta}-\left.Q_{\mu}(\alpha)\right|_{\alpha=0} Z_{\mu 0}, \\
-\left.U_{\mu}(\alpha)\right|_{\alpha=\alpha_{\mathrm{M}}}=F_{\sigma}-\left.Q_{\mu}(\alpha)\right|_{\alpha=\alpha_{\mathrm{M}}} Z_{\mu 0}
\end{array}\right\}
$$

Where $Z_{\mu 0}$ is the internal magnetic resistance of MDS sources.

It should be noted that the sign of the magnetic voltage between the concentric ferromagnetic cores 1 and 2 on both sides of the magnetic neutral $M-M^{\prime}$, which when $Z_{\mu 01}=Z_{\mu 02}=Z_{\mu 0}$ coinciding with the geometric neutral $0-0$ ', varies (the direction of the scattering fluxes that branch off from one concentric core to the other along the annular gap $\delta$ also varies) Figure 1). Therefore, on the left-hand side of the second equation of system (7) $U_{\mu}(\alpha)$ enters with the sign “-”.

Substituting in (6) the values of magnetic fluxes and magnetic stresses corresponding to the boundary conditions, we obtain the following system of algebraic equations:

$$
\left.\begin{array}{l}
\left(1-\frac{\gamma Z_{\mu 0}}{2 Z_{\mu \mathrm{I}}}\right) A_{1}+\left(1+\frac{\gamma Z_{\mu 0}}{2 Z_{\mu \mathrm{n}}}\right) A_{2}=F_{6}, \\
\left(1+\frac{\gamma Z_{\mu 0}}{2 Z_{\mu \mathrm{T}}}\right) e^{\gamma \alpha_{\mathrm{M}}} A_{1}+\left(1-\frac{\gamma Z_{\mu 0}}{2 Z_{\mu \mathrm{I}}}\right) e^{-\gamma \alpha_{\mathrm{M}}} A_{2}=-F_{\theta} \cdot
\end{array}\right\}
$$

Solving the system of equations (8) with respect to the integration constants $A_{1}$ and $A_{2}$, we obtain:

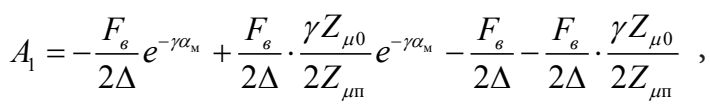

$$
A_{2}=\frac{F_{b}}{2 \Delta}-\frac{F_{b}}{2 \Delta} \cdot \frac{\gamma Z_{\mu 0}}{2 Z_{\mu п}}+\frac{F_{s}}{2 \Delta} e^{\gamma \alpha_{\mathrm{M}}}+\frac{F_{\theta}}{2 \Delta} \cdot \frac{\gamma Z_{\mu 0}}{2 Z_{\mu п}} e^{\gamma \alpha_{\mathrm{M}}}
$$

where

$\Delta=\left(1+\frac{\gamma^{2} Z_{\mu 0}^{2}}{4 Z_{\mu п}^{2}} \operatorname{sh}\left(\gamma \alpha_{\mathrm{M}}\right)\right)+\frac{\gamma Z_{\mu 0}}{Z_{\mu п}} \operatorname{ch}\left(\gamma \alpha_{\mathrm{M}}\right)$ 
Substituting the values found $A_{1}$ and $A_{2}$ in expressions (5) and (6), we have

$$
\begin{aligned}
& U_{\mu}(\alpha)=\frac{F_{6}}{\Delta}\left\{\operatorname{sh}\left[\gamma\left(\alpha_{\mathrm{M}}-\alpha\right)\right]-\operatorname{sh}(\gamma \alpha)\right\}+ \\
& \frac{F_{\theta}}{\Delta} \cdot \frac{\gamma Z_{\mu 0}}{2 Z_{\mu \mathrm{n}}}\left\{\operatorname{ch}\left[\gamma\left(\alpha_{\mathrm{M}}-\alpha\right)\right]-\operatorname{ch}(\gamma \alpha)\right\}
\end{aligned}
$$

$$
\begin{aligned}
& Q_{\mu}(\alpha)=\frac{\gamma F_{s}}{2 Z_{\mu \mathrm{m}} \Delta}\left\{\left\{\operatorname{ch}\left[\gamma\left(\alpha_{\mathrm{M}}-\alpha\right)\right]+\operatorname{ch}(\gamma \alpha)\right\}+\right. \\
& \frac{\gamma Z_{\mu 0}}{2 Z_{\mu \mathrm{I}}}\left\{\operatorname{sh}\left[\gamma\left(\alpha_{\mathrm{M}}-\alpha\right)\right]+\operatorname{sh}(\gamma \alpha)\right\}
\end{aligned}
$$

We transform (11) and (12) using the following hyperbolic trigonometry formulas [3]:

$$
\begin{aligned}
& \operatorname{sh} x \pm \operatorname{sh} y=2 \operatorname{sh}\left[\frac{1}{2}(x \pm y)\right] \operatorname{ch}\left[\frac{1}{2}(x \mp y)\right], \\
& \operatorname{ch} x+\operatorname{ch} y=2 \operatorname{ch}\left[\frac{1}{2}(x+y)\right] \operatorname{ch}\left[\frac{1}{2}(x-y)\right], \\
& \operatorname{ch} x-\operatorname{ch} y=2 \operatorname{sh}\left[\frac{1}{2}(x+y)\right] \operatorname{sh}\left[\frac{1}{2}(x-y)\right] . \\
& U_{\mu}(\alpha)=\frac{F_{\sigma}}{2 \Delta}\left[\operatorname{ch}\left(\frac{1}{2} \gamma \alpha_{\mathrm{M}}\right)+\frac{\gamma Z_{\mu 0}}{2 Z_{\mu \mathrm{m}}} \operatorname{sh}\left(\frac{1}{2} \gamma \alpha_{\mathrm{M}}\right)\right] \\
& \operatorname{sh}\left[\gamma\left(\frac{1}{2} \alpha_{\mathrm{M}}-\alpha\right)\right], \\
& Q_{\mu}(\alpha)=\frac{\gamma F_{\sigma}}{Z_{\mu \mathrm{m}} \Delta}\left[\operatorname{ch}\left(\frac{1}{2} \gamma \alpha_{\mathrm{M}}\right)+\frac{\gamma Z_{\mu 0}}{2 Z_{\mu \mathrm{m}}} \operatorname{sh}\left(\frac{1}{2} \gamma \alpha_{\mathrm{M}}\right)\right] \\
& \operatorname{ch}\left[\gamma\left(\frac{1}{2} \alpha_{\mathrm{M}}-\alpha\right)\right] .
\end{aligned}
$$

Denoting $\lambda \alpha_{\mathrm{M}}=\beta, \mathrm{k}_{0}=\frac{Z_{\mu 0}}{2 Z_{\mu \mathrm{m}} \alpha_{\mathrm{M}}}, \alpha^{*}=\frac{\alpha}{\alpha_{\mathrm{M}}}$ we
ewrite (14) and (15):

$$
\begin{aligned}
& U_{\mu}\left(\alpha^{*}\right)=\frac{F_{6}}{2 \Delta}\left[\operatorname{ch}\left(\frac{1}{2} \beta\right)+\beta K_{0} \operatorname{sh}\left(\frac{1}{2} \beta\right)\right] \\
& \operatorname{sh}\left[\beta\left(\frac{1}{2}-\alpha^{*}\right)\right],
\end{aligned}
$$

$$
\begin{aligned}
& Q_{\mu}\left(\alpha^{*}\right)=\frac{\beta F_{\varepsilon}}{Z_{\mu \mathrm{I}} \alpha_{\mathrm{M}} \Delta}\left[\operatorname{ch}\left(\frac{1}{2} \beta\right)+\beta K_{0} \operatorname{sh}\left(\frac{1}{2} \beta\right)\right] \\
& \operatorname{ch}\left[\beta\left(\frac{1}{2}-\alpha^{*}\right)\right],
\end{aligned}
$$

where $\beta$ - the damping coefficient of the magnetic flux along the magnetic circuit.

Expressions (16) and (17) are mathematical models of the magnetic circuits of the developed magnetoelastic force sensors, taking into account the distributed nature of the magnetic resistances of concentric ferromagnetic cores and the magnetic capacitance between these coaxially located cores.

To facilitate the analysis of these expressions, we proceed in relative units:

$$
\begin{gathered}
U_{\mu}^{*}\left(\alpha^{*}\right)=\frac{U_{\mu}\left(\alpha^{*}\right)}{U_{\mu}(0)}=\frac{\operatorname{sh}\left[\beta \frac{1}{2}-\alpha^{*}\right]}{\operatorname{sh}\left(\frac{1}{2} \beta\right)}, \\
Q_{\mu}^{*}\left(\alpha^{*}\right)=\frac{Q_{\mu}\left(\alpha^{*}\right)}{Q_{\mu}(0)}=\frac{\operatorname{ch}\left[\gamma \frac{1}{2}-\alpha^{*}\right]}{\operatorname{ch}\left(\frac{1}{2} \beta\right)},
\end{gathered}
$$

here $U_{\mu}(0)$ and $Q_{\mu}(0)$ maximum values respectively $U_{\mu}\left(\alpha^{*}\right)$ and $Q_{\mu}\left(\alpha^{*}\right)$ at $\alpha^{*}=0$

In Fig. 3 and Fig. 4 the dependency curves $U_{\mu}\left(\alpha^{*}\right)=f\left(\alpha^{*}\right)$ and $Q_{\mu}\left(\alpha^{*}\right)=f\left(\alpha^{*}\right)$ for different values $\beta$.

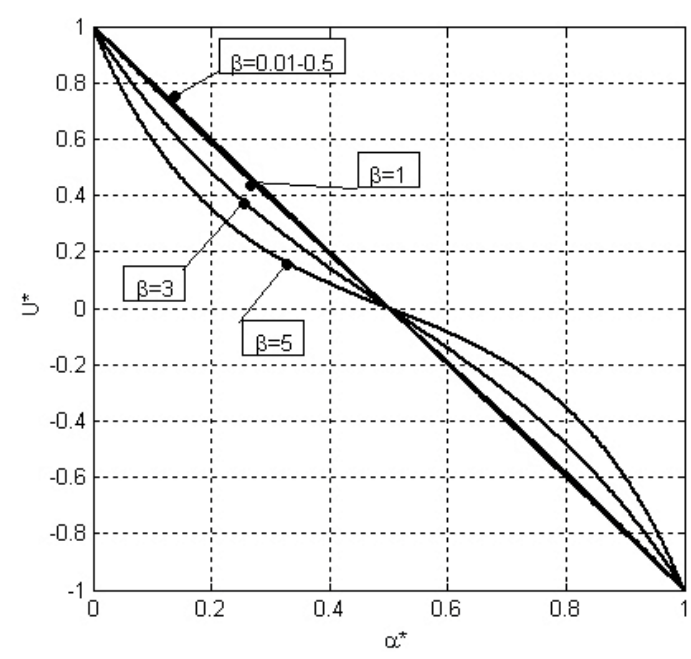

Fig. 3. Dependence curves $U_{\mu}\left(\alpha^{*}\right)=f\left(\alpha^{*}\right)$ for different values of $\beta$. 
Analysis of expressions (16) and (17), as well as curves based on them, shows that with increasing magnetic flux damping along the magnetic circuit $\beta$, the degree of nonlinearity of the change in the magnetic voltage and the inconstancy of the magnetic flux along the magnetic circuit increases.

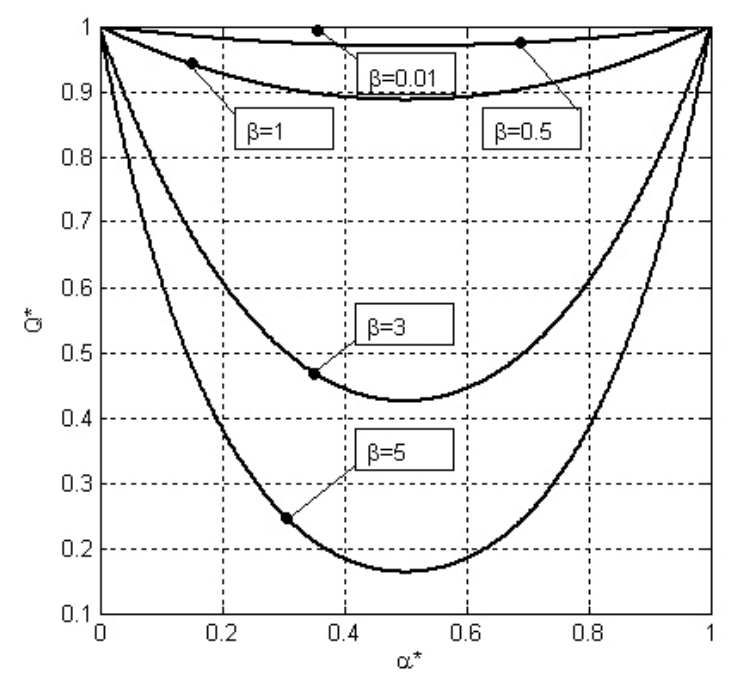

Fig. 4. Dependence curves for different values of $\beta$.

Thus, in the article mathematical models of magnetic circuits of the developed magnetoelastic force sensors are developed, taking into account the distributed nature of the magnetic resistances of concentric ferromagnetic cores and magnetic capacitance between these coaxially located cores. It is shown that as the magnetic flux attenuation coefficient increases along the magnetic circuit $\beta$, the degree of nonlinearity of the change in the magnetic voltage and the inconstancy of the magnetic flux along the magnetic circuit increases.

\section{REFERENCES}

[1] Patent Uzbekistan. №IAP 04866. Magnetoelastic force sensor / S.F. Amirov, Juraeva K.K., Nazirova Z.G. et al. // Rasmiy Ahborotnoma. - 2014. - №4.

[2] Positive decision to grant a patent for an invention under application No. IAP 2015 0228. Magnetoelastic force sensor / Amirov S.F., Juraeva K.K., Turdybekov K.K., Boltaev O.T., Faizullaev J.S. Declared June, 2015.

[3] Theoretical foundations of electrical engineering: In 3 volumes. Textbook for high schools. -4 th ed. / K.S. Demirchyan, L.R. Neiman, N.V. Korovkin, V.L. Chechurin. - St. Petersburg: Peter, 2006. - Volume 1 (320 p.), Volume 2 (576 p.), Volume 3 (377 p.).

[4] Amirov S.F, Sulliev A.Kh., Balgaev N.E. A Brief Overview of the Methods for Calculating Magnetic Circuits with Distributed Parameters. // Journal of TASHTU "Problems of Energy and Resource Saving” - Tashkent, 2010.- № 1/2 - P. 195-202.

[5] Zaripov M.F. Converters with distributed parameters for automation and information-measuring equipment. - Moscow: Energy, 1969 - 176 p.

[6] Konyukhov NE, Mednikov FM, Nechaevsky M.L. Electromagnetic sensors of mechanical quantities // - Moscow: Mechanical Engineering, 1987. - 256 p.

[7] Probotiuk V.V. Mathematical model of the magnetoelastic transducer and its harmonic representation: Thesis for a scientific degree of Cand.Tech.Sci. - Tyumen: Tashkent State Oil and Gas University, 2005. -112 p. 